EPJ Web of Conferences 88, 00009 (2015)

DOI: $10.1051 /$ epjconf/ 20158800009

(C) Owned by the authors, published by EDP Sciences - SIF, 2015

\title{
Comparison of radial flow effects on partitions of multifragmenting sources formed in symmetric and asymmetric central collisions
}

\author{
J.D. Frankland, D. Gruyer, E. Bonnet and A. Chbihi \\ for the INDRA/E613 COLLABORATION
}

Grand Accélérateur National d'Ions Lourds, B.P. 55027, Boulevard Henri Bécquerel, F-14076 Caen, France

\begin{abstract}
The role of collective radial expansion in determining multifragmentation partition properties has previously been explored by comparing different-sized sources of the same excitation energy per nucleon formed by very different reaction mechanisms: excited quasi-projectiles from semi-peripheral $\mathrm{Au}+\mathrm{Au}$ collisions and quasi-fused sources from central $\mathrm{Xe}+\mathrm{Sn}$ collisions. New data has been obtained with INDRA on ${ }^{181} \mathrm{Ta}+{ }^{66} \mathrm{Zn}$ collisions in order to allow comparison of $\mathrm{Xe}+\mathrm{Sn}$ data with quasi-fused systems having the same mass, charge and excitation energy per nucleon formed in mass-asymmetric reactions, for which a smaller initial compression is expected. Preliminary results confirm that, ceteris paribus, smaller radial flow leads to less fragments with more asymmetric partitions.
\end{abstract}

\section{Introduction}

Multifragmentation has long been associated with the predicted liquid-gas coexistence region in the nuclear matter phase diagram at sub-critical temperatures and sub-saturation densities [1,2]. Central collisions between heavy nuclei can lead to formation of quasi-fused systems composed of 
hot compressed nuclear matter, which subsequently expands and clusterizes at low densities [3-5]. Such reactions have been extensively studied with INDRA using the near mass-symmetric Xe+Sn collisions [6-10]. As the dominant decay mode for hot nuclei with excitation energies in the range $3 \sim 10 \mathrm{AMeV}$, multifragmentation can also be studied using excited quasi-projectiles formed in semi-peripheral collisions [11] or in hadronnucleus reactions [12]. Comparison of these three production modes has shown that the onset of compression-expansion in central collisions (above available centre-of-mass energies of $\sim 5 \mathrm{AMeV}$ ) leads to a larger collective radial motion of fragments than by thermal pressure alone [13]. Although the proportion of radial flow energy never exceeds $20 \%$ of the total in this excitation energy range, it was shown to play a crucial role in determining fragment partitions [14]: for a given excitation energy and source size, whatever the production mode, the amount of radial flow fixes the mean fragment multiplicity, which in turn determines the partition properties. To put it simply, more flow means more fragments, with more symmetric (similar-sized fragments) partitions.

In order to further test our understanding of the link between radial flow and multifragment partitions, new experimental data have been obtained in 2011 with INDRA at GANIL using a mass-asymmetric reaction with the same total mass and charge as $\mathrm{Xe}+\mathrm{Sn}:{ }^{181} \mathrm{Ta}+{ }^{66} \mathrm{Zn}$. The bombarding energies used in the experiment, 32 and $39 \mathrm{AMeV}$, were chosen in order to give the same available centre of mass energies as for the existing $\mathrm{Xe}+\mathrm{Sn}$ data at 25 and $32 \mathrm{AMeV}$. For an asymmetric entrance channel we expect less initial compression of the quasi-fused (QF) systems formed in central collisions, due to the smaller geometric overlap between the two incident nuclei, and hence less radial flow of the produced fragments. The aim of the experiment is to compare the partitions and radial flow of fragments produced by QF sources of same size and excitation energy formed in the two reactions.

\section{Experimental details}

The $\mathrm{Xe}+\mathrm{Sn}$ data were measured during the fifth INDRA campaign, details can be found in [15]. In the new experiment, beams of ${ }^{181} \mathrm{Ta}^{57+}$ at $39 \mathrm{AMeV}$ and ${ }^{181} \mathrm{Ta}^{52+}$ at $32 \mathrm{AMeV}$ were delivered by the coupled cyclotrons of GANIL, with intensities of $\sim 10^{6}-10^{7}$ pps. They impinged on a $99 \%$ isotopically-pure self-supported ${ }^{66} \mathrm{Zn}$ target of $350 \mu \mathrm{g} / \mathrm{cm}^{2}$ thickness. The complete INDRA multidetector was used in its standard con- 

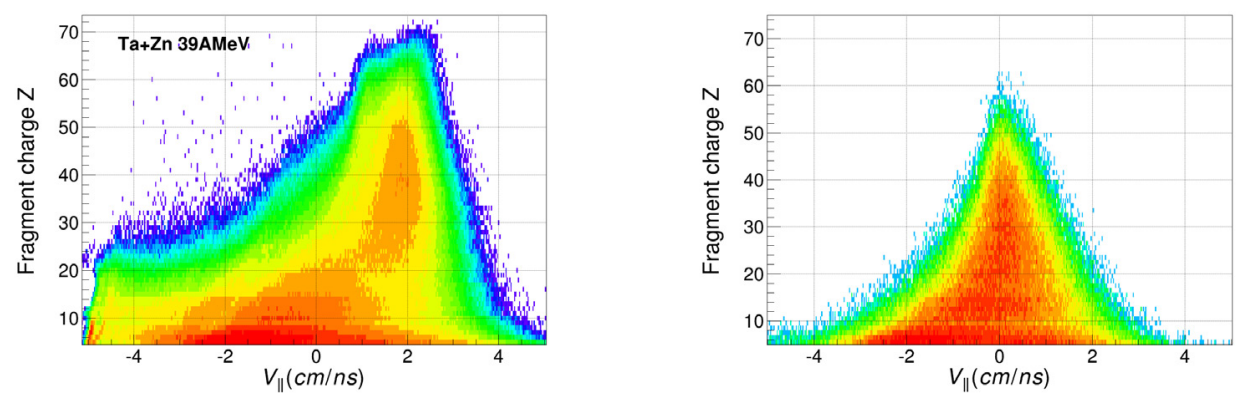

Figure 1: Measured fragment charge as a function of centre-of-mass longitudinal velocity for well-measured (see text) ${ }^{181} \mathrm{Ta}+{ }^{66} \mathrm{Zn} 39 \mathrm{AMeV}$ collisions without (left) and with (right) a cut $\theta_{\text {flow }} \geq 70^{\circ}$ to select $\mathrm{QF}$ sources.

figuration $[16,17]$, with the most forward $\operatorname{ring}\left(\theta_{\text {lab }}=2^{\circ}-3^{\circ}\right)$ of phoswiches replaced by $\mathrm{Si}(300 \mu \mathrm{m})-\mathrm{CsI}(\mathrm{Tl})$ telescopes, and using $150 \mu \mathrm{m}$ silicon wafers on rings $4-7\left(\theta_{\text {lab }}=7^{\circ}-27^{\circ}\right)$ instead of the standard $300 \mu \mathrm{m}$ detectors. Due to mechanical problems with the aluminized mylar windows, only the most forward ionisation chambers (rings $2-6, \theta_{\text {lab }}=3^{\circ}-27^{\circ}$ ) operated correctly throughout the experiment. Despite this drawback, thanks to the use of inverse kinematics most of the produced fragments had sufficient kinetic energy to punch through the silicon detectors on the forward rings of INDRA, allowing a near-complete reconstruction of the reaction kinematics.

\section{Comparison of symmetric and asymmetric reac- tions}

\subsection{Selection of quasi-fused (QF) sources}

Quasi-fused multifragmenting systems were selected using the angle characterizing the global orientation of the fragment momentum distribution, $\theta_{\text {flow }}$, calculated event-by-event from the fragment kinetic energy tensor (see $[13,18]$ for details). This procedure requires a complete reconstruction of the event kinematics, therefore in the following we consider only events for which the sum of all correctly identified and calibrated charged products' atomic numbers, $Z_{\text {tot }}=\sum_{i} Z_{i}$, is at least 90 , to be compared with the total available charge of $104(\mathrm{Xe}+\mathrm{Sn})$ or $103(\mathrm{Ta}+\mathrm{Zn})$. An additional cut was made on the total pseudo-momentum of the reconstructed events, $0.8 \leq \sum_{i} Z_{i} v_{i} / Z_{\text {proj }} v_{\text {proj }} \leq 1.1$.

Figure 1 shows the effect of a cut $\theta_{\text {flow }} \geq 70^{\circ}$ on the fragment charge- 
Table 1: Characteristics of selected QF sources as a function of available centre-ofmass energy $\epsilon_{\mathrm{c} . \mathrm{m}}$. for (S)ymmetric and (A)symmetric reactions: mean multiplicity of light charged particles $(Z \leq 2), M_{\mathrm{lcp}}$; mean total charge of fragments $(Z \geq 5)$, $Z_{\text {bound }}$; mean fragment multiplicity, $M_{Z \geq 5}$; mean charge of the largest fragment of each event, $Z_{\max }$; normalised mean relative velocity between all fragments, $\beta_{\text {rel }}^{(N)}$; $Z_{\max }$ distribution fitting parameters, $\eta$ and $R$ (see text).

\begin{tabular}{ccccccccc}
\hline & & $M_{\text {lcp }}$ & $Z_{\text {bound }}$ & $M_{Z \geq 5}$ & $Z_{\text {max }}$ & $\beta_{\text {rel }}^{(N)}$ & $\eta$ & $R$ \\
\hline$\sim 6 A \mathrm{MeV}$ & $\mathrm{S}$ & 15 & 69 & 4.0 & 32 & 0.39 & 0.33 & -0.3 \\
& $\mathrm{~A}$ & 14 & 71 & 3.3 & 37 & 0.33 & 0.79 & 0.6 \\
\hline \multirow{2}{*}{$\sim A \mathrm{MeV}$} & $\mathrm{S}$ & 18 & 63 & 4.6 & 25 & 0.48 & 0.21 & -0.6 \\
& $\mathrm{~A}$ & 17 & 65 & 3.8 & 31 & 0.39 & 0.54 & 0.1 \\
\hline
\end{tabular}

velocity correlations for the ${ }^{181} \mathrm{Ta}+{ }^{66} \mathrm{Zn} 39 \mathrm{AMeV}$ reaction. The ensemble of well-measured events (Figure 1, left) are clearly dominated by the decay of projectile- and target-like nuclei, accompanied by many intermediate mass fragments produced at intermediate velocities. This is a typical picture of highly-dissipative heavy-ion reactions at Fermi energies [19,20]. On the other hand, it can be seen (Figure 1, right) that the $\theta_{\text {flow }} \geq 70^{\circ}$ cut effectively selects events with quasi-symmetric charge-velocity correlations in the fragments' centre-of-mass frame, typical of the decay products of a welldefined QF source. In order to make the least biased comparison possible, we have applied exactly the same cuts to the four reactions considered here in order to obtain the samples of $\mathrm{QF}$ sources which we will now examine in more detail.

\section{$3.2 \quad$ Source properties \& partitions}

Table 1 presents the properties of the four QF source samples obtained according to the procedure presented in 3.1. As shown in the first column they can be considered as two pairs of symmetric/asymmetric reactions for available centre-of-mass energies of $\epsilon_{\text {c.m. }} \sim 6$ and $\epsilon_{\text {c.m. }} \sim 8 \mathrm{AMeV}$. In the current state of data reduction (see Sec.2), calorimetric methods applied to the ${ }^{181} \mathrm{Ta}+{ }^{66} \mathrm{Zn}$ data would lead to a systematic under-estimation of the QF source excitation energies because no calibration is available for particles emitted at backward angles. However, the mean light charged particle multiplicity, $M_{\mathrm{lcp}}$, includes particles emitted over the full $4 \pi$ solid angle and can therefore be used as a rough comparator for $\epsilon^{*}$. Table 1 shows that for each of the two available energies, $M_{\mathrm{lcp}}$ is the same within one 

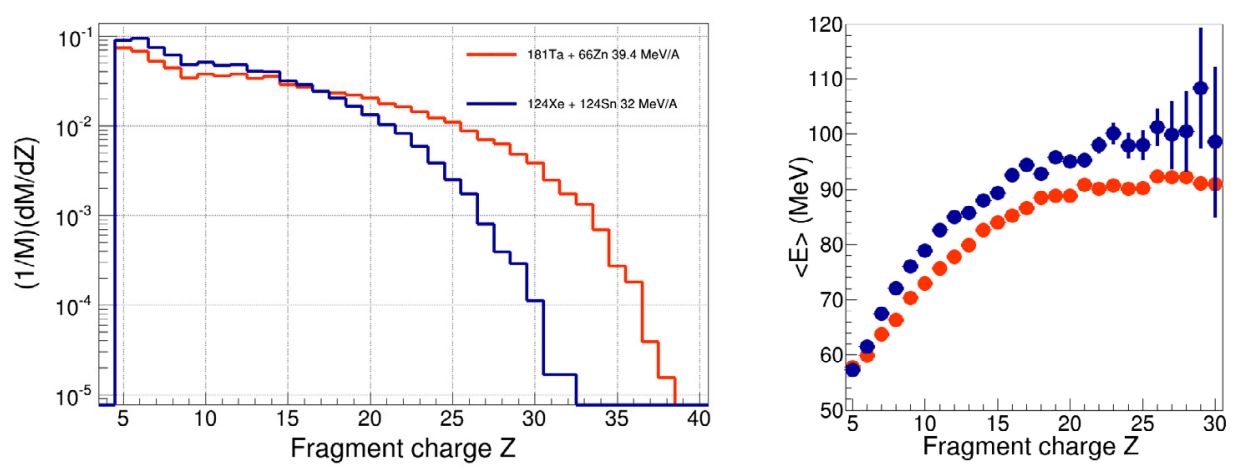

Figure 2: Comparison between QF sources produced in symmetric (blue) and asymmetric (orange) reactions at $\epsilon_{\text {c.m. }} \sim 8 \mathrm{AMeV}$, excluding the largest fragment of each event, $Z_{\max }$ : (left) fragment differential multiplicity distributions; (right) mean kinetic energy of fragments in their centre-of-mass frame as a function of fragment charge.

unit for symmetric and asymmetric reactions ${ }^{1}$, indicating that very similar excitation energies are reached.

Now let us consider the fragment partitions. The mean charge bound in fragments, $Z_{\text {bound }}=\left\langle\sum_{Z_{i} \geq 5} Z_{i}\right\rangle$, is very nearly independent (within $\sim 3 \%$ ) of the entrance channel asymmetry, whereas mean fragment multiplicities are $\sim 20 \%$ larger for the symmetric systems. Consequently, the mean charge of the largest fragment produced in each event, $Z_{\max }=\max _{i}\left\{Z_{i}\right\}$, is significantly larger for both asymmetric reactions, and the differential multiplicity distributions of the remaining fragments (see Figure 2 (left)) show a systematic trend: at each available energy, the production of heavier fragments is enhanced at the expense of lighter ones.

\subsection{Radial flow effects}

We now want to address the question of the amount of radial expansion present in each reaction. The most obvious starting point is the fragment kinetic energies. The mean kinetic energy of each fragment can be approximated as the sum of thermal, Coulomb, and radial flow contributions. To first order, we can assume the same mean thermal contribution for QF sources with the same thermal excitation energy, as we deduced from the measured light charged particle multiplicities (see 3.2). Similarly, we can

\footnotetext{
${ }^{1}$ Let us recall that the $\mathrm{Xe}+\mathrm{Sn}$ entrance channel has one extra proton compared to ${ }^{181} \mathrm{Ta}+{ }^{66} \mathrm{Zn}$.
} 
consider that for a fragment of given charge, $Z$, produced by QF sources of same total charge $Z_{\text {bound }}$, the average Coulomb contribution will be the same. Thus, the systematically larger mean kinetic energy for symmetric as compared to asymmetric reactions observed in Figure 2 (right) is a first indication of larger radial expansion of QF sources formed in symmetric reactions. It should be noted that here we have excluded the heaviest fragment, $Z_{\max }$, from the average for each $Z$, as its mean energy is systematically lower than the others, as seen in $[6,7]$.

A trivial Coulomb effect still remains when comparing fragment kinetic energies, due to the differences in partitions between symmetric and asymmetric reactions. This can be removed by using a simple normalization of the mean fragment relative velocities which takes into account, event by event, the differences in multiplicity and mean charge of the fragments [13]:

$$
<\beta_{\text {rel }}^{(N)}>=100 \frac{<\beta_{i}-\beta_{j}>_{i>j}}{\sqrt{<Z>\left(Z_{\text {bound }}-<Z>\right)}}
$$

Values are given in Table 1. Larger values of $\left\langle\beta_{\text {rel }}^{(N)}>\right.$ are obtained for the symmetric reaction at each available energy, by an amount which is comparable to the differences between $\mathrm{QF}$ and QP sources in this energy range reported in [13]. Such a non-trivial increase of the mean fragment relative velocities points to the presence of larger radial flow for QF sources formed in symmetric rather than asymmetric reactions at the same available/excitation energy.

Another, indirect, method to gauge the importance of radial flow in multifragmentation reactions was suggested in [21], where it was shown that the $Z_{\max }$ distributions in central $\mathrm{Xe}+\mathrm{Sn}$ collisions evolve with bombarding energy in a manner similar to the largest cluster size distribution in numerical simulations of the Smoluchowski equations for irreversible aggregation in finite systems: for short clusterisation times, $s_{\max }$ is an extremal order parameter characterised by the Gumbel distribution; over long timescales $s_{\max }$ becomes an additive order parameter characterised by the Gaussian distribution; for intermediate timescales (around the critical gelation time), the $s_{\max }$ distribution can be approximated by an admixture of the two asymptotic forms,

$$
P\left(s_{\text {max }}\right)=\eta P_{\text {Gauss }}+(1-\eta) P_{\text {Gumbel }}
$$

Fits to the experimental $Z_{\max }$ distributions for the four reactions have been achieved, and examples for the ${ }^{181} \mathrm{Ta}+{ }^{66} \mathrm{Zn}$ data are shown in Figure 3. As in [21], it can be seen that Eq. 2 nicely reproduces the non-trivial shapes of these distributions. The corresponding values of $\eta$ are given in 

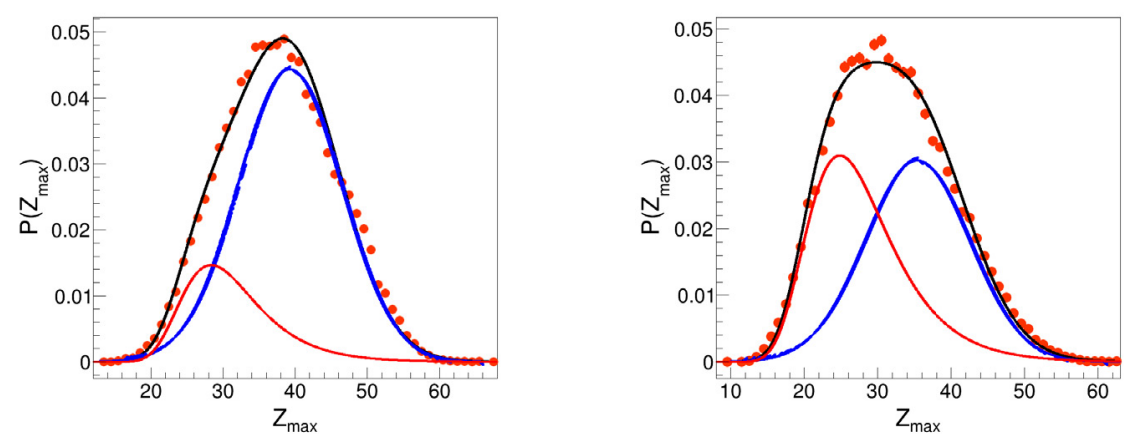

Figure 3: (Colour online) $Z_{\max }$ distributions (points) for QF sources produced in ${ }^{181} \mathrm{Ta}+{ }^{66} \mathrm{Zn}$ reactions at (left) $32 \mathrm{AMeV}$ and (right) $39 \mathrm{AMeV}$. The black curves are fits to data using Eq. 2; the two component distributions are shown in red (Gumbel) and blue (Gauss) respectively.

Table 1. They are systematically larger for the asymmetric reactions at the two available energies, meaning that the Gaussian/additive behaviour is more dominant, indicative of longer clusterisation times. Another way of representing this is with the quantity $R=2 \eta-1$ which is positive for long timescales, and negative for faster, more explosive clusterisation. Again, the values of $R$ given in Table 1 are consistent with faster clusterisation for the symmetric reactions, which we associate with the presence of larger radial flow.

\section{Conclusions}

In this contribution we presented a preliminary comparison of multifragmenting quasi-fused/single sources formed in symmetric and asymmetric reactions with almost identical mass, charge and available energy. New data on ${ }^{181} \mathrm{Ta}+{ }^{66} \mathrm{Zn}$ reactions in inverse kinematics measured with INDRA in 2011 were presented for the first time, and compared to existing datasets for $\mathrm{Xe}+\mathrm{Sn}$ reactions obtained in 2001. Although data reduction for ${ }^{181} \mathrm{Ta}+{ }^{66} \mathrm{Zn}$ is not complete, the use of inverse kinematics allows to obtain high statistics for very well-measured central collisions using complete identification and calibration of only the forward part of the detector.

For each of the two centre-of-mass available energies of $\epsilon_{\text {c.m. }} \sim 6$ and $\epsilon_{\mathrm{c} . \mathrm{m} .} \sim 8 \mathrm{AMeV}$, mean light charged particle multiplicities for the two reactions have unit difference, suggesting that QF source excitation energies are independent of the entrance channel asymmetry. Similarly, the total charge 
bound in fragments is very nearly the same for symmetric and asymmetric reactions. On the other hand, we observed a clear difference in the way this bound charge is partitioned: at each $\epsilon_{\text {c.m. }}$, less fragments are produced on average in the asymmetric reactions, giving more asymmetric partitions with a larger average $Z_{\max }$. We then showed evidence for smaller collective radial energy for the asymmetric reactions, by comparison of mean fragment center-of-mass kinetic energies and the Coulomb-corrected fragmentfragment relative velocities. A striking difference in the form of the $Z_{\max }$ distributions was also seen, which evidences longer clusterisation times in the asymmetric reactions due to reduced radial flow.

These preliminary results therefore confirm the conclusions of [14] that for a given excitation energy per nucleon and source size, it is the amount of radial flow which determines the fragment multiplicity and partition properties. This invalidates one of the basic hypotheses of most statistical models of multifragmentation, in which partitions and flow are decoupled in the calculation of the statistical weights [22]. On the other hand, such a coupling occurs naturally in dynamical models capable of simulating the entire history of the formation and multifragmentation of hot compressed nuclear blobs [5,23]. Comparisons with such models are underway and will allow to better understand the fundamentally dynamical nature of the multifragmentation process.

\section{References}

[1] Bertsch G. and Siemens P. J., Physics Letters B 1269 (1983).

[2] Jaqaman H., Mekjian A. and Zamick L., Physical Review C 29, 2067 (1984).

[3] Chomaz P., Colonna M. and Randrup J., Physics Reports 389, 263 (2004).

[4] Rizzo J., Colonna M. and Ono A., Physical Review C 76, 024611 (2007).

[5] Bonnet E., Colonna M. et al., Physical Review C 89034608 (2014).

[6] Marie N., Laforest R. et al., Physics Letters B 391, 15 (1997).

[7] Frankland J. D., Borderie B. et al. Nuclear Physics A689, 940 (2001).

[8] Hudan S., Chbihi A. at al., Physical Review C67, 064613 (2003). 
[9] Le Neindre N., Bonnet E. et al., Nuclear Physics A795, 47 (2007).

[10] Piantelli S., Borderie B. et al., Nuclear Physics A809, 111 (2008).

[11] Srivastava B. K., Scharenberg R. P. et al., Physical Review C 65, 05461 (2002).

[12] Viola V. E., Kwiatkowski K. et al., Physics Reports 434, 1 (2006).

[13] Bonnet E., Borderie B. et al., Nuclear Physics 816, 1 (2009).

[14] Bonnet E., Borderie B. et al., Physical Review Letters 105, 142701 (2010).

[15] Moisan F. G., Galichet E. et al., Physical Review C 86, 044617 (2012).

[16] Pouthas J., Borderie B. et al., Nucl. Instr. Meth. Phys. Res.A 357, 418 (1995).

[17] Pouthas J., Bertaut A. et al., Nucl. Instr. Meth. Phys. Res.A 369, 222 (1996).

[18] Frankland J. D., Bacri C.-O. et al., Nuclear Physics A 689, 905 (2001).

[19] Plagnol E., Lukasik J. et al., Physical Review C 61, 014606 (1999).

[20] Filippo E., Pagano A. et al., Physical Review C 71 (2005).

[21] Gruyer D., Frankland J. D. et al., Physical Review Letters 110, 172701 (2013).

[22] Bondorf J. P., Botvina A. S. et al., Physics Reports 257, 133 (1995).

[23] Napolitani P. and Colonna M., Physics Letters B 726, 382 (2013). 